\title{
A Proposal to the Study of Contractions in Quasi-Metric Spaces
}

\author{
Hamed H. Alsulami, ${ }^{1}$ Erdal Karapınar, ${ }^{1,2}$ \\ Farshid Khojasteh, ${ }^{3}$ and Antonio-Francisco Roldán-López-de-Hierro ${ }^{4}$ \\ ${ }^{1}$ Nonlinear Analysis and Applied Mathematics Research Group (NAAM), King Abdulaziz University, Jeddah, Saudi Arabia \\ ${ }^{2}$ Department of Mathematics, Atilim University, Incek, 06836 Ankara, Turkey \\ ${ }^{3}$ Department of Mathematics, Arak Branch, Islamic Azad University, Arak, Iran \\ ${ }^{4}$ Department of Mathematics, University of Jaén, Campus las Lagunillas s/n, 23071 Jaén, Spain
}

Correspondence should be addressed to Erdal Karapınar; erdalkarapinar@yahoo.com

Received 28 March 2014; Accepted 10 July 2014; Published 6 August 2014

Academic Editor: Janusz Brzdęk

Copyright (C) 2014 Hamed H. Alsulami et al. This is an open access article distributed under the Creative Commons Attribution License, which permits unrestricted use, distribution, and reproduction in any medium, provided the original work is properly cited.

We investigate the existence and uniqueness of a fixed point of an operator via simultaneous functions in the setting of complete quasi-metric spaces. Our results generalize and improve several recent results in literature.

\section{Introduction and Preliminaries}

One of the attractive research subjects in the fixed point theory is the investigation of the existence and uniqueness of (common) fixed point of various operators in the setting of quasi-metric space. Very recently, Jleli and Samet [1] and Samet et al. [2] reported that G-metrics, introduced by Mustafa and Sims [3], can be deduced from quasi-metrics by taking $q(x, y)=G(x, y, y)$. Consequently, the authors in $[1,2]$ proved that several fixed point results in the setting of $G$-metric spaces can be deduced from the corresponding theorems in the context of quasi-metric spaces. The importance of these results follows from the simplicity of construction of quasi-metric despite the notion of $G$-metric.

In this paper, we investigate the existence and uniqueness of a fixed point of operators via simultaneous functions, defined by Khojasteh et al. [4], in the setting of complete quasi-metric spaces. We also observed that several existing results can be concluded from our main results. We also show that some result in the context of $G$-metric spaces can be deduced from the corresponding theorems in the framework of quasi-metric spaces.

For the sake of completeness, we recollect basic notions, definitions, and fundamental results. Let $A, B \subseteq X$ be two nonempty subsets of a set $X$ and let $T: A \rightarrow B$ be a mapping.
A point $x \in X$ is called a fixed point of the mapping $T$ if $T x=$ $x$.

Definition 1. Let $X$ be a nonempty set and let $q: X \times X \rightarrow$ $[0,+\infty)$ be a given function which satisfies

(1) $q(x, y)=0$ if and only if $x=y$;

(2) $q(x, y) \leq q(x, z)+q(z, y)$ for any points $x, y, z \in X$.

Then $q$ is called a quasi-metric and the pair $(X, q)$ is called a quasi-metric space.

It is evident that any metric space is a quasi-metric space, but the converse is not true in general. Now, we recall convergence and completeness on quasi-metric spaces.

Definition 2. Let $(X, q)$ be a quasi-metric space and let $\left\{x_{n}\right\}$ be a sequence in $X$ and $x \in X$. The sequence $\left\{x_{n}\right\}$ converges to $x$ if

$$
\lim _{n \rightarrow \infty} q\left(x_{n}, x\right)=\lim _{n \rightarrow \infty} q\left(x, x_{n}\right)=0 .
$$

Remark 3. A convergent sequence in a quasi-metric space has a unique limit. 
Remark 4. If $\left\{x_{n}\right\}$ converges to $x$ in a quasi-metric space $(X, q)$, then

$$
\lim _{n \rightarrow \infty} q\left(x_{n}, y\right)=q(x, y), \quad \forall y \in X
$$

In other words, $q$ is a continuous mapping on its first argument. This property follows from $q\left(x_{n}, y\right) \leq q\left(x_{n}, x\right)+$ $q(x, y)$ and $q(x, y) \leq q\left(x, x_{n}\right)+q\left(x_{n}, y\right)$. Therefore,

$$
\begin{array}{r}
q(x, y)-q\left(x, x_{n}\right) \leq q\left(x_{n}, y\right) \leq q\left(x_{n}, x\right)+q(x, y) \\
\forall n \in \mathbb{N} .
\end{array}
$$

Definition 5 (see, e.g., $[1,2])$. Let $(X, q)$ be a quasi-metric space and let $\left\{x_{n}\right\}$ be a sequence in $X$. We say that $\left\{x_{n}\right\}$ is left-Cauchy if, for every $\varepsilon>0$, there exists a positive integer $N=N(\varepsilon)$ such that $q\left(x_{n}, x_{m}\right)<\varepsilon$ for all $n \geq m>N$.

Definition 6 (see, e.g., $[1,2])$. Let $(X, q)$ be a quasi-metric space and let $\left\{x_{n}\right\}$ be a sequence in $X$. We say that $\left\{x_{n}\right\}$ is right-Cauchy if, for every $\varepsilon>0$, there exists a positive integer $N=N(\varepsilon)$ such that $q\left(x_{n}, x_{m}\right)<\varepsilon$ for all $m \geq n>N$.

Definition 7 (see, e.g., $[1,2])$. Let $(X, q)$ be a quasi-metric space and let $\left\{x_{n}\right\}$ be a sequence in $X$. We say that $\left\{x_{n}\right\}$ is Cauchy if, for every $\varepsilon>0$, there exists a positive integer $N=N(\varepsilon)$ such that $q\left(x_{n}, x_{m}\right)<\varepsilon$ for all $m, n>N$.

Remark 8. A sequence $\left\{x_{n}\right\}$ in a quasi-metric space is Cauchy if and only if it is left-Cauchy and right-Cauchy.

Definition 9 (see, e.g., $[1,2])$. Let $(X, q)$ be a quasi-metric space. We say that

(1) $(X, q)$ is left-complete if each left-Cauchy sequence in $X$ is convergent;

(2) $(X, q)$ is right-complete if each right-Cauchy sequence in $X$ is convergent;

(3) $(X, q)$ is complete if each Cauchy sequence in $X$ is convergent.

\section{Simulation Functions}

The notion of simulation function was introduced by Khojasteh et al. in [4].

Definition 10 (see [4]). A simulation function is a mapping $\zeta:[0, \infty) \times[0, \infty) \rightarrow \mathbb{R}$ satisfying the following conditions:

$\left(\zeta_{1}\right) \zeta(0,0)=0$

$\left(\zeta_{2}\right) \zeta(t, s)<s-t$ for all $t, s>0$;

$\left(\zeta_{3}\right)$ if $\left\{t_{n}\right\},\left\{s_{n}\right\}$ are sequences in $(0, \infty)$ such that $\lim _{n \rightarrow \infty} t_{n}=\lim _{n \rightarrow \infty} s_{n}>0$ then

$$
\limsup _{n \rightarrow \infty} \zeta\left(t_{n}, s_{n}\right)<0 .
$$

Let $\mathscr{Z}$ be the family of all simulation functions $\zeta$ : [0, $\infty) \times$ $[0, \infty) \rightarrow \mathbb{R}$
Before presenting our main fixed point results using simulation functions, we show a wide range of examples to highlight their potential applicability to the field of fixed point theory. In the following results, the mapping $\zeta$ is defined from $[0, \infty) \times[0, \infty)$ into $\mathbb{R}$.

Definition 11 (Khan et al. [5]). An altering distance function is a continuous, nondecreasing mapping $\phi:[0, \infty) \rightarrow[0, \infty)$ such that $\phi^{-1}(\{0\})=\{0\}$.

Example 12. Let $\phi$ and $\psi$ be two altering distance functions such that $\psi(t)<t \leq \phi(t)$ for all $t>0$. Then the mapping

$$
\zeta_{1}(t, s)=\psi(s)-\phi(t), \quad \forall t, s \in[0, \infty)
$$

is a simulation function.

If, in the previous example, $\phi(t)=t$ and $\psi(t)=\lambda t$ for all $t \geq 0$, where $\lambda \in[0,1)$, then we obtain the following particular case of simulation function:

$$
\zeta_{B}(t, s)=\lambda s-t, \quad \forall t, s \in[0, \infty)
$$

Example 13. If $\varphi:[0, \infty) \rightarrow[0, \infty)$ is a lower semicontinuous function such that $\varphi^{-1}(0)=\{0\}$ and we define $\zeta_{R}$ : $[0, \infty) \times[0, \infty) \rightarrow \mathbb{R}$ by

$$
\zeta_{R}(t, s)=s-\varphi(s)-t, \quad \forall s, t \in[0, \infty),
$$

then $\zeta_{R}$ is a simulation function.

If, in the previous example, $\varphi$ is continuous, we deduce the following case.

Example 14. If $\varphi:[0, \infty) \rightarrow[0, \infty)$ is a continuous function such that $\varphi(t)=0 \Leftrightarrow t=0$ and we define

$$
\zeta_{R}(t, s)=s-\varphi(s)-t, \quad \forall s, t \in[0, \infty),
$$

then $\zeta_{R}$ is a simulation function.

Example 15. Let $f, g:[0, \infty) \rightarrow(0, \infty)$ be two continuous functions with respect to each variable such that $f(t, s)>$ $g(t, s)$ for all $t, s>0$ and define

$$
\zeta(t, s)=s-\frac{f(t, s)}{g(t, s)} t, \quad \forall t, s \in[0, \infty) .
$$

Then $\zeta$ is a simulation function.

Example 16. If $\varphi:[0, \infty) \rightarrow[0,1)$ is a function such that $\limsup _{t \rightarrow r^{+}} \varphi(t)<1$ for all $r>0$ and we define

$$
\zeta_{T}(t, s)=s \varphi(s)-t, \quad \forall s, t \in[0, \infty),
$$

then $\zeta_{T}$ is a simulation function.

Example 17. If $\eta:[0, \infty) \rightarrow[0, \infty)$ is an upper semicontinuous mapping such that $\eta(t)<t$ for all $t>0$ and $\eta(0)=0$ and we define

$$
\zeta_{B W}(t, s)=\eta(s)-t, \quad \forall s, t \in[0, \infty),
$$

then $\zeta_{B W}$ is a simulation function. 
Example 18. If $\phi:[0, \infty) \rightarrow[0, \infty)$ is a function such that $\int_{0}^{\varepsilon} \phi(u) d u$ exists and $\int_{0}^{\varepsilon} \phi(u) d u>\varepsilon$, for each $\varepsilon>0$, and we define

$$
\zeta_{K}(t, s)=s-\int_{0}^{t} \phi(u) d u, \quad \forall s, t \in[0, \infty),
$$

then $\zeta_{K}$ is a simulation function.

Example 19. Let $h:[0, \infty) \times[0, \infty) \rightarrow[0, \infty)$ be a function such that $h(t, s)<1$ for all $t, s>0$ and $\lim _{\sup } \rightarrow \infty h\left(t_{n}, s_{n}\right)<$ 1 provided that $\left\{t_{n}\right\}$ and $\left\{s_{n}\right\} \subset(0,+\infty)$ are two sequences such that $\lim _{n \rightarrow \infty} t_{n}=\lim _{n \rightarrow \infty} s_{n}>0$, and we define

$$
\zeta_{U}(t, s)=s h(t, s)-t, \quad \forall s, t \in[0, \infty),
$$

and then $\zeta_{U}$ is a simulation function.

The following results are more theoretical.

Proposition 20. Let $\eta:[0, \infty) \times[0, \infty) \rightarrow \mathbb{R}$ be a function such that $\eta(0,0)=0$ and there exists $\zeta \in \mathscr{Z}$ verifying that $\eta(t, s) \leq \zeta(t, s)$ for all $s, t \geq 0$. Then $\eta \in \mathscr{Z}$.

Proof. For all $t, s>0, \eta(t, s) \leq \zeta(t, s)<s-t$. If $\left\{t_{n}\right\}$ and $\left\{s_{n}\right\}$ are sequences in $(0, \infty)$ such that $\lim _{n \rightarrow \infty} t_{n}=\lim _{n \rightarrow \infty} s_{n}=\delta>$ 0 , then $\lim \sup _{n \rightarrow \infty} \eta\left(t_{n}, s_{n}\right) \leq \lim \sup _{n \rightarrow \infty} \zeta\left(t_{n}, s_{n}\right)<0$.

Proposition 21. Let $\left\{\eta_{i}\right\}_{i \in \mathbb{N}} \subset \mathscr{Z}$. Then the following statements hold.

$$
\begin{aligned}
& \text { (a) For each } k \in \mathbb{N} \text {, the function } \eta_{(k)}^{\min }: \mathbb{R} \times \mathbb{R} \rightarrow \mathbb{R} \text { defined } \\
& \quad \text { by } \\
& \eta_{(k)}^{\min }(t, s)=\min \left\{\eta_{1}(t, s), \eta_{2}(t, s), \ldots, \eta_{k}(t, s)\right\} \quad \forall t, s \geq 0
\end{aligned}
$$

is a simulation function (i.e., $\eta_{(k)}^{\min } \in \mathscr{Z}$ for any $k \in \mathbb{N}$ ).

(b) For each $k \in \mathbb{N}$, the function $\overline{\eta_{(k)}}: \mathbb{R} \times \mathbb{R} \rightarrow \mathbb{R}$ defined by

$$
\overline{\eta_{(k)}}(t, s)=\frac{1}{k} \sum_{i=1}^{k} \eta_{i}(t, s), \quad \forall t, s \geq 0
$$

is a simulation function (i.e., $\overline{\eta_{(k)}} \in$ for any $k \in \mathbb{N}$ ).

Proof. Since $\eta_{(k)}^{\min }(t, s) \leq \eta_{1}(t, s)$ for all $t, s>0$, the conclusion (a) is a direct consequence of Proposition 20. Next, we prove the conclusion (b). Let $k \in \mathbb{N}$ be given. It is obvious that $\overline{\eta_{(k)}}(t, s)<s-t$ for all $s, t>0$ because

$$
\overline{\eta_{(k)}}(t, s)=\frac{1}{k} \sum_{i=1}^{k} \eta_{i}(t, s)<\frac{1}{k} \sum_{i=1}^{k}(s-t)=s-t .
$$

Let $\left\{t_{n}\right\},\left\{s_{n}\right\} \subset(0,+\infty)$ be two sequences such that $\lim _{n \rightarrow \infty} t_{n}=\lim _{n \rightarrow \infty} s_{n}=\delta>0$. For any $n \in \mathbb{N}$, we have

$$
\limsup _{n \rightarrow \infty} \overline{\eta_{(k)}}\left(t_{n}, s_{n}\right)=\frac{1}{k} \sum_{i=1}^{k} \limsup _{n \rightarrow \infty} \eta_{i}\left(t_{n}, s_{n}\right)<0 .
$$

\section{Main Results}

In this section we use simulation functions to present a very general kind of contractions on quasi-metric spaces, and we prove related existence and uniqueness fixed point theorems.

Definition 22. Let $(X, q)$ be a quasi-metric space. We will say that a self-mapping $T: X \rightarrow X$ is a $\mathscr{Z}$-contraction if there exists $\zeta \in \mathscr{Z}$ such that

$$
\zeta(q(T x, T y), q(x, y)) \geq 0, \quad \forall x, y \in X .
$$

For clarity, we will use the term $\mathscr{Z}_{q}$-contraction when we want to highlight that $T$ is a $\mathscr{Z}$-contraction on a quasi-metric space involving the quasi-metric $q$. In such a case, we will say that $T$ is a $\mathscr{Z}_{q}$-contraction with respect to $\zeta$.

Next, we observe some useful properties of $\mathscr{Z}_{q}$ contractions in the context of quasi-metric spaces.

Remark 23. By axiom $\left(\zeta_{3}\right)$, it is clear that a simulation function must verify $\zeta(r, r)<0$ for all $r>0$. Consequently, if $T$ is a $\mathscr{Z}_{q}$-contraction with respect to $\zeta \in \mathscr{Z}_{q}$, then

$$
q(T x, T y) \neq q(x, y), \quad \forall \text { distinct } x, y \in X .
$$

In other words, if $T$ is a $\mathscr{Z}_{q}$-contraction, then it cannot be an isometry.

We will prove that if a $\mathscr{Z}_{q}$-contraction has a fixed point, then it is unique.

Lemma 24. If a $\mathscr{Z}_{q}$-contraction in a quasi-metric space has a fixed point, then it is unique.

Proof. Let $(X, q)$ be a quasi-metric space and let $T: X \rightarrow X$ be a $\mathscr{Z}_{q}$-contraction with respect to $\zeta \in \mathscr{Z}$. We are reasoning by contradiction. Suppose that there are two distinct fixed points $u, v \in X$ of the mapping $T$. Then $q(u, v)>0$. By (18), we have

$$
0 \leq \zeta(q(T u, T v), q(u, v))=\zeta(q(u, v), q(u, v)),
$$

which is a contradiction due to Remark 23.

Inspired by Browder and Petryshyn's paper [6], we will characterize the notions of asymptotically right-regularity and asymptotically left-regularity for a self-mapping $T$ in the context of quasi-metric space $(X, q)$.

Definition 25. We will say that a self-mapping $T: X \rightarrow X$ on a quasi-metric space $(X, q)$ is

(i) asymptotically right-regular at a point $x \in X$ if $\lim _{n \rightarrow \infty} q\left(T^{n} x, T^{n+1} x\right)=0 ;$

(ii) asymptotically left-regular at a point $x \in X$ if $\lim _{n \rightarrow \infty} q\left(T^{n+1} x, T^{n} x\right)=0$

(iii) asymptotically regular if it is both asymptotically right-regular and asymptotically left-regular.

Now, we show that a $\mathscr{Z}_{q}$-contraction is asymptotically regular at every point of $X$. 
Lemma 26. Every $\mathscr{Z}_{q^{-c o n t r a c t i o n}}$ on a quasi-metric space is asymptotically regular.

Proof. Let $x$ be an arbitrary point of a quasi-metric space $(X, q)$ and let $T: X \rightarrow X$ be a $\mathscr{E}_{q}$-contraction with respect to $\zeta \in \mathscr{Z}$. If there exists some $p \in \mathbb{N}$ such that $T^{p} x=T^{p-1} x$, then $y=T^{p-1} x$ is a fixed point of $T$; that is, $T y=y$. Consequently, we have that $T^{n} y=y$ for all $n \in \mathbb{N}$, so

$$
\begin{aligned}
q\left(T^{n} x, T^{n+1} x\right) & =q\left(T^{n-p+1} T^{p-1} x, T^{n-p+2} T^{p-1} x\right) \\
& =q\left(T^{n-p+1} y, T^{n-p+2} y\right)=q(y, y)=0,
\end{aligned}
$$

for sufficient large $n \in \mathbb{N}$. Thus, we conclude that

$$
\lim _{n \rightarrow \infty} q\left(T^{n} x, T^{n+1} x\right)=0 .
$$

Similarly, $\lim _{n \rightarrow \infty} q\left(T^{n+1} x, T^{n} x\right)=0$, so $T$ is asymptotically regular at $x$. On the contrary, suppose that $T^{n} x \neq T^{n-1} x$ for all $n \in \mathbb{N}$; that is,

$$
q\left(T^{n} x, T^{n-1} x\right)>0, \quad \forall n \in \mathbb{N} .
$$

On what follows, from (18) and $\left(\zeta_{2}\right)$, we have that, for all $n \in$ $\mathbb{N}$,

$$
\begin{aligned}
0 & \leq \zeta\left(q\left(T^{n+1} x, T^{n} x\right), q\left(T^{n} x, T^{n-1} x\right)\right) \\
& <q\left(T^{n} x, T^{n-1} x\right)-q\left(T^{n+1} x, T^{n} x\right) .
\end{aligned}
$$

In particular,

$$
q\left(T^{n+1} x, T^{n} x\right)<q\left(T^{n} x, T^{n-1} x\right), \quad \forall n \in \mathbb{N} .
$$

The above inequality yields that $\left\{q\left(T^{n} x, T^{n-1} x\right)\right\}$ is a monotonically decreasing sequence of nonnegative real numbers. Thus, there exists $r \in[0, \infty)$ such that $\lim _{n \rightarrow \infty} q\left(T^{n} x\right.$, $\left.T^{n+1} x\right)=r \geq 0$. We will prove that $r=0$. Suppose, on the contrary, that $r>0$. Since $T$ is $\mathscr{E}_{q}$-contraction with respect to $\zeta \in \mathscr{E}_{q}$, by $\left(\zeta_{3}\right)$, we have

$$
0 \leq \limsup _{n \rightarrow \infty} \zeta\left(q\left(T^{n+1} x, T^{n} x\right), q\left(T^{n} x, T^{n-1} x\right)\right)<0,
$$

which is a contradiction. Thus, $r=0$ and this proves that $\lim _{n \rightarrow \infty} q\left(T^{n} x, T^{n+1} x\right)=0$. Hence, $T$ is an asymptotically right-regular mapping at $x$. Similarly, it can be demonstrated that $T$ is asymptotically left-regular at $x$.

Given a self-mapping $T: X \rightarrow X$, a sequence $\left\{x_{n}\right\} \subseteq X$ is called a Picard sequence of $T$ (or generated by $T)$ if $x_{n}=T x_{n-1}$ for all $n \in \mathbb{N}$.

Remark 27. In the proof of the previous result we have proved that if $T: X \rightarrow X$ is a $\mathscr{E}_{q}$-contraction on a quasi-metric space $(X, q)$ and $\left\{x_{n}=T^{n-1} x_{1}\right\}$ is a Picard sequence of $T$, then either there exists $n_{0} \in \mathbb{N}$ such that $x_{n_{0}}$ is a fixed point of $T$ (i.e., $x_{n_{0}+1}=T x_{n_{0}}=x_{n_{0}}$ ) or

$$
\begin{array}{r}
0<q\left(T^{n+1} x, T^{n} x\right)<q\left(T^{n} x, T^{n-1} x\right), \\
0<q\left(T^{n} x, T^{n+1} x\right)<q\left(T^{n-1} x, T^{n} x\right), \\
\forall n \in \mathbb{N} .
\end{array}
$$

Now, we show that every Picard sequence $\left\{x_{n}\right\}$ generated by a $\mathscr{Z}_{q}$-contraction is always bounded.

Lemma 28. Let $(X, q)$ be a quasi-metric space and let $T$ :

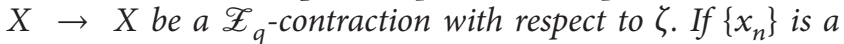
Picard sequence generated by $T$, then $\left\{q\left(x_{n}, x_{m}\right): n, m \in \mathbb{N}\right\}$ is bounded.

Proof. Let $x_{0} \in X$ be arbitrary and let $\left\{x_{n}\right\}$ be defined iteratively by $x_{n+1}=T x_{n}$ for all $n \geq 0$. If there exists some $n \geq 0$ and $p \geq 1$ such that $x_{n+p}=x_{n}$, then the set $\left\{x_{n}: n \in \mathbb{N}\right\}$ is finite, so it is bounded. Hence, assume that $x_{n+p} \neq x_{n}$ for all $n \geq 0$ and $p \geq 1$. In this case, by Remark 27, we have that

$$
\begin{array}{r}
0<q\left(x_{n+1}, x_{n}\right)<q\left(x_{n}, x_{n-1}\right), \\
0<q\left(x_{n}, x_{n+1}\right)<q\left(x_{n-1}, x_{n}\right), \\
\forall n \in \mathbb{N} .
\end{array}
$$

Notice that by Lemma 26,

$$
\lim _{n \rightarrow \infty} q\left(x_{n+1}, x_{n}\right)=\lim _{n \rightarrow \infty} q\left(x_{n}, x_{n+1}\right)=0 .
$$

In particular, there exists $n_{0} \in \mathbb{N}$ such that

$$
q\left(x_{n+1}, x_{n}\right)<1, \quad q\left(x_{n}, x_{n+1}\right)<1, \quad \forall n \geq n_{0} .
$$

We will prove that $\left\{x_{n}: n \in \mathbb{N}\right\}$ is bounded reasoning by contradiction. We distinguish between right and left boundedness. Suppose that the set

$$
D=\left\{q\left(x_{m}, x_{n}\right): m>n\right\}
$$

is not bounded. Then we can find $n_{1}>n_{0}$ such that $q\left(x_{n_{1}}, x_{n_{0}}\right)>1$. If $n_{1}$ is the smallest natural number, greater than $n_{0}$, verifying this property, then we can suppose that

$$
q\left(x_{p}, x_{n_{0}}\right) \leq 1, \quad \forall p \in\left\{n_{0}, n_{0}+1, \ldots, n_{1}-1\right\} .
$$

Again, as $D$ is not bounded, there exists $n_{2}>n_{1}$ such that

$$
\begin{array}{r}
q\left(x_{n_{2}}, x_{n_{1}}\right)>1, \quad q\left(x_{p}, x_{n_{1}}\right) \leq 1, \\
\forall p \in\left\{n_{1}, n_{1}+1, \ldots, n_{2}-1\right\} .
\end{array}
$$

Repeating this process, there exists a partial subsequence $\left\{x_{n_{k}}\right\}$ of $\left\{x_{n}\right\}$ such that, for all $k \geq 1$,

$$
\begin{array}{r}
q\left(x_{n_{k+1}}, x_{n_{k}}\right)>1, \quad q\left(x_{p}, x_{n_{k}}\right) \leq 1, \\
\forall p \in\left\{n_{k}, n_{k}+1, \ldots, n_{k+1}-1\right\} .
\end{array}
$$


Therefore, by the triangular inequality, we have that, for all $k$,

$$
\begin{aligned}
1<q\left(x_{n_{k+1}}, x_{n_{k}}\right) & \leq q\left(x_{n_{k+1}}, x_{n_{k+1}-1}\right)+q\left(x_{n_{k+1}-1}, x_{n_{k}}\right) \\
& \leq q\left(x_{n_{k+1}}, x_{n_{k+1}-1}\right)+1 .
\end{aligned}
$$

Letting $k \rightarrow \infty$ in (35) and using (29) we obtain

$$
\lim _{k \rightarrow \infty} q\left(x_{n_{k+1}}, x_{n_{k}}\right)=1
$$

By (28), we have $q\left(x_{n_{k+1}}, x_{n_{k}}\right) \leq q\left(x_{n_{k+1}-1}, x_{n_{k}-1}\right)$. Therefore using the triangular inequality we obtain

$$
\begin{aligned}
1 & <q\left(x_{n_{k+1}}, x_{n_{k}}\right) \leq q\left(x_{n_{k+1}-1}, x_{n_{k}-1}\right) \\
& \leq q\left(x_{n_{k+1}-1}, x_{n_{k}}\right)+q\left(x_{n_{k}}, x_{n_{k}-1}\right) \\
& \leq 1+q\left(x_{n_{k}}, x_{n_{k}-1}\right) .
\end{aligned}
$$

Letting $k \rightarrow \infty$ and using (29) we obtain

$$
\lim _{k \rightarrow \infty} q\left(x_{n_{k+1}-1}, x_{n_{k}-1}\right)=1 .
$$

Owing to the fact that $T$ is a $\mathscr{Z}_{q}$-contraction with respect to $\zeta \in \mathscr{Z}_{q}$, we deduce from $\left(\zeta_{3}\right)$ that, for all $k$,

$$
\begin{aligned}
0 & \leq \limsup _{k \rightarrow \infty} \zeta\left(q\left(T x_{n_{k+1}-1}, T x_{n_{k}-1}\right), q\left(x_{n_{k+1}-1}, x_{n_{k}-1}\right)\right) \\
& =\limsup _{k \rightarrow \infty} \zeta\left(q\left(x_{n_{k+1}}, x_{n_{k}}\right), q\left(x_{n_{k+1}-1}, x_{n_{k}-1}\right)\right)<0,
\end{aligned}
$$

which is a contradiction. This proves that $D=\left\{q\left(x_{m}, x_{n}\right)\right.$ : $m>n\}$ is bounded. Similarly, it can be proved that $D^{\prime}=$ $\left\{q\left(x_{m}, x_{n}\right): m<n\right\}$ is also bounded. Therefore, the set $\left\{q\left(x_{m}, x_{n}\right): m, n \in \mathbb{N}\right\}$ is bounded.

In the next theorem we prove the existence of fixed point of a $\mathscr{Z}_{q}$-contraction.

Theorem 29. Every $\mathscr{Z}$-contraction on a complete quasi-metric space has a unique fixed point. In fact, every Picard sequence converges to its unique fixed point.

Proof. Let $(X, q)$ be a complete quasi-metric space and let $T$ : $X \rightarrow X$ be a $\mathscr{Z}_{q}$-contraction with respect to $\zeta$. Take $x_{0} \in$ $X$ and consider the Picard sequence $\left\{x_{n}=T^{n} x_{0}\right\}_{n \geq 0}$. If $\left\{x_{n}\right\}$ contains a fixed point of $T$, the proof is finished. In other case, Lemma 26 and Remark 27 guarantee that

$$
\begin{gathered}
0<q\left(x_{n+1}, x_{n}\right)<q\left(x_{n}, x_{n-1}\right), \\
0<q\left(x_{n}, x_{n+1}\right)<q\left(x_{n-1}, x_{n}\right), \\
\forall n \in \mathbb{N} . \\
\lim _{n \rightarrow \infty} q\left(x_{n+1}, x_{n}\right)=\lim _{n \rightarrow \infty} q\left(x_{n}, x_{n+1}\right)=0 .
\end{gathered}
$$

We are going to show that $\left\{x_{n}\right\}$ is a left Cauchy sequence. For this purpose, taking into account that Lemma 28 guarantees that $\left\{q\left(x_{m}, x_{n}\right): m, n \in \mathbb{N}\right\}$ is bounded, we can consider the sequence $\left\{C_{n}\right\} \subset[0, \infty)$ given by

$$
C_{n}=\sup \left(\left\{q\left(x_{i}, x_{j}\right): i \geq j \geq n\right\}\right), \quad \forall n \in \mathbb{N} .
$$

It is clear that the sequence $\left\{C_{n}\right\}$ is a monotonically nonincreasing sequence of nonnegative real numbers. Therefore, it is convergent; that is, there exists $C \geq 0$ such that $\lim _{n \rightarrow \infty} C_{n}=C$. Let us show that $C=0$ reasoning by contradiction. If $C>0$ then, by definition of $C_{n}$, for every $k \in \mathbb{N}$ there exists $n_{k}, m_{k} \in \mathbb{N}$ such that $m_{k}>n_{k} \geq k$ and

$$
C_{k}-\frac{1}{k}<q\left(x_{m_{k}}, x_{n_{k}}\right) \leq C_{k}
$$

Hence,

$$
\lim _{k \rightarrow \infty} q\left(x_{m_{k}}, x_{n_{k}}\right)=C
$$

By using (40) and the triangular inequality, we have, for all $k$,

$$
\begin{aligned}
q\left(x_{m_{k}}, x_{n_{k}}\right) & \leq q\left(x_{m_{k}-1}, x_{n_{k}-1}\right) \\
& \leq q\left(x_{m_{k}-1}, x_{m_{k}}\right)+q\left(x_{m_{k}}, x_{n_{k}}\right)+q\left(x_{n_{k}}, x_{n_{k}-1}\right) .
\end{aligned}
$$

Letting $k \rightarrow \infty$ in the above inequality and using (41) and (44), we derive that

$$
\lim _{k \rightarrow \infty} q\left(x_{m_{k}-1}, x_{n_{k}-1}\right)=C .
$$

Due to fact that $T$ is a $\mathscr{Z}_{q}$-contraction with respect to $\zeta \in \mathscr{Z}_{q}$ and by using $\left(\zeta_{3}\right),(18),(44)$, and (46), we have

$$
\begin{aligned}
0 & \leq \limsup _{k \rightarrow \infty} \zeta\left(q\left(T x_{m_{k}}, T x_{n_{k}}\right), q\left(x_{m_{k}}, x_{n_{k}}\right)\right) \\
& =\limsup _{k \rightarrow \infty} \zeta\left(q\left(x_{m_{k}-1}, x_{n_{k}-1}\right), q\left(x_{m_{k}}, x_{n_{k}}\right)\right)<0,
\end{aligned}
$$

which is a contradiction. This contradiction concludes that $C=0$ and, hence, $\left\{x_{n}\right\}$ is a left Cauchy sequence. Similarly, it can be proved that $\left\{x_{n}\right\}$ is a right Cauchy sequence. Therefore, $\left\{x_{n}\right\}$ is a Cauchy sequence. Since $(X, q)$ is a complete quasimetric space, there exists $u \in X$ such that $\lim _{n \rightarrow \infty} x_{n}=u$.

We will show that the point $u$ is a fixed point of $T$ reasoning by contradiction. Suppose that $T u \neq u$; that is, $q(u, T u)>0$. By Remark 4,

$$
\lim _{n \rightarrow \infty} q\left(T x_{n}, T u\right)=\lim _{n \rightarrow \infty} q\left(x_{n+1}, T u\right)=q(u, T u)>0 .
$$

Therefore, there is $n_{0} \in \mathbb{N}$ such that

$$
q\left(T x_{n}, T u\right)>0, \quad \forall n \geq n_{0} .
$$

In particular, $T x_{n} \neq T u$. This also means that $x_{n} \neq u$ for all $n \geq n_{0}$. As $q\left(T x_{n}, T u\right)>0$ and $q\left(x_{n}, u\right)>0$, axiom $\left(\zeta_{2}\right)$ and property (18) imply that, for all $n \geq n_{0}$,

$$
0 \leq \zeta\left(q\left(T x_{n}, T u\right), q\left(x_{n}, u\right)\right)<q\left(x_{n}, u\right)-q\left(T x_{n}, T u\right) .
$$


In particular, $0 \leq q\left(T x_{n}, T u\right) \leq q\left(x_{n}, u\right)$ for all $n \geq n_{0}$, which means that

$$
\lim _{n \rightarrow \infty} q\left(x_{n+1}, T u\right)=\lim _{n \rightarrow \infty} q\left(T x_{n}, T u\right)=0 .
$$

Similarly, it can be proved that $\lim _{n \rightarrow \infty} q\left(T u, x_{n+1}\right)=0$. Therefore, $\left\{x_{n}\right\}$ converges, at the same time, to $u$ and to $T u$. By the unicity of the limit, $u=T u$, which contradicts $T u \neq u$. As a consequence, $u$ is a fixed point of $T$. Notice that the uniqueness of the fixed point follows from Lemma 24.

Next, we show a variety of cases in which Theorem 29 can be applied. Firstly, we mention the analog of the celebrated Banach contraction principle [7] in quasi-metric spaces.

Corollary 30 (see, e.g., [1]). Let $(X, q)$ be a complete quasimetric space and let $T: X \rightarrow X$ be a mapping such that

$$
q(T x, T y) \leq \lambda q(x, y), \quad \forall x, y \in X,
$$

where $\lambda \in[0,1)$. Then $T$ has a unique fixed point in $X$.

Proof. The result follows from Theorem 29 taking into account that $T$ is a $\mathscr{E}_{q}$-contraction with respect to $\zeta_{B} \in \mathscr{Z}$, where $\zeta_{B}$ is defined by $\zeta_{B}(t, s)=\lambda s-t$ for all $s, t \in[0, \infty)$ (see (6)).

The following example shows that the above theorem is a proper generalization of the analog of Banach contraction principle.

Example 31. Let $\alpha, \beta, k \in(0,1)$ be such that $\alpha \leq k$. Let $X=$ $[0,1]$ and $q: X \times X \rightarrow[0, \infty)$ be a function defined by

$$
q(x, y)= \begin{cases}x-y, & \text { if } x \geq y, \\ \beta(y-x), & \text { if } x<y .\end{cases}
$$

Then $(X, q)$ is a complete quasi-metric space (but it is not a metric space). Consider the mapping $T: X \rightarrow X$ defined as $T x=\alpha x$ for all $x \in X$. It is clear that it is a $\mathscr{E}_{q}$-contraction with respect to $\zeta \in \mathscr{Z}$, where

$$
\zeta(t, s)=k s-t, \quad \forall t, s \in[0, \infty) .
$$

Indeed, if $x \geq y$, then $T x \geq T y$. Hence, we get that

$$
\begin{aligned}
\zeta & (q(T x, T y), q(x, y)) \\
& =\zeta(q(\alpha x, \alpha y), q(x, y))=\zeta(\alpha(x-y), x-y) \\
& =k(x-y)-\alpha(x-y)=(k-\alpha)(x-y) \geq 0 .
\end{aligned}
$$

If $x<y$ then $T x<T y$. Hence, we get that

$$
\begin{aligned}
\zeta( & (T x, T y), q(x, y)) \\
& =\zeta(q(\alpha x, \alpha y), q(x, y))=\zeta(\beta(\alpha y-\alpha x), \beta(y-x)) \\
& =k \beta(y-x)-\beta(\alpha y-\alpha x)=\beta(k-\alpha)(y-x) \geq 0 .
\end{aligned}
$$

Notice that all conditions in Theorem 29 are satisfied and $T$ has a unique fixed point, which is $x=0$.
In the following corollaries we obtain some known and some new results in fixed point theory via simulation functions.

Corollary 32 (Rhoades type). Let $(X, q)$ be a complete quasimetric space and let $T: X \rightarrow X$ be a mapping satisfying the following condition:

$$
q(T x, T y) \leq q(x, y)-\varphi(q(x, y)) \quad \forall x, y \in X,
$$

where $\varphi:[0, \infty) \rightarrow[0, \infty)$ is a lower semicontinuous function and $\varphi^{-1}(0)=\{0\}$. Then $T$ has a unique fixed point in $X$.

Proof. The result follows from Theorem 29 taking into account that $T$ is a $\mathscr{E}_{q}$-contraction with respect to $\zeta_{R} \in \mathscr{Z}$, where $\zeta_{R}$ is defined by $\zeta_{R}(t, s)=s-\varphi(s)-t$ for all $s, t \in[0, \infty)$ (see Example 13).

Remark 33. Note that Rhoades assumed in [8] that the function $\varphi$ was continuous and nondecreasing and it verified $\lim _{t \rightarrow \infty} \varphi(t)=\infty$. In Corollary 32, we replace these conditions by the lower semicontinuity of $\varphi$, which is a weaker condition. Therefore, our result is stronger than Rhoades' original version.

Corollary 34. Let $(X, q)$ be a complete quasi-metric space and let $T: X \rightarrow X$ be a mapping. Suppose that for every $x, y \in X$,

$$
q(T x, T y) \leq \varphi(q(x, y)) q(x, y)
$$

for all $x, y \in X$, where $\varphi:[0,+\infty) \rightarrow[0,1)$ is a function such that $\lim \sup _{t \rightarrow r^{+}} \varphi(t)<1$ for all $r>0$. Then $T$ has a unique fixed point.

Proof. The result follows from Theorem 29 taking into account that $T$ is a $\mathscr{E}_{q}$-contraction with respect to $\zeta_{T} \in \mathscr{Z}$, where $\zeta_{T}$ is defined by $\zeta_{T}(t, s)=s \varphi(s)-t$ for all $s, t \in[0, \infty)$ (see Example 16).

Corollary 35. Let $(X, q)$ be a complete quasi-metric space and let $T: X \rightarrow X$ be a mapping. Suppose that, for every $x, y \in X$,

$$
q(T x, T y) \leq \eta(q(x, y))
$$

for all $x, y \in X$, where $\eta:[0,+\infty) \rightarrow[0,+\infty)$ is an upper semicontinuous mapping such that $\eta(t)<t$ for all $t>0$ and $\eta(0)=0$. Then $T$ has a unique fixed point.

Proof. The result follows from Theorem 29 taking into account that $T$ is a $\mathscr{Z}_{q}$-contraction with respect to $\zeta_{B W} \in \mathscr{Z}$, where $\zeta_{B W}$ is defined by $\zeta_{B W}(t, s)=\eta(s)-t$ for all $s, t \in[0, \infty)$ (see Example 17).

Corollary 36. Let $(X, q)$ be a complete quasi-metric space and let $T: X \rightarrow X$ be a mapping satisfying the following condition:

$$
\int_{0}^{q(T x, T y)} \phi(t) d t \leq q(x, y) \quad \forall x, y \in X,
$$

where $\phi:[0, \infty) \rightarrow[0, \infty)$ is a function such that $\int_{0}^{\epsilon} \phi(t) q t$ exists and $\int_{0}^{\epsilon} \phi(t) d t>\epsilon$, for each $\epsilon>0$. Then $T$ has a unique fixed point in $X$. 
Proof. The result follows from Theorem 29 taking into account that $T$ is a $\mathscr{Z}_{q}$-contraction with respect to $\zeta_{K} \in \mathscr{Z}$, where $\zeta_{K}$ is defined by

$$
\zeta_{K}(t, s)=s-\int_{0}^{t} \phi(u) q u \quad \forall s, t \in[0, \infty)
$$

(see Example 18).

Corollary 37. Let $(X, q)$ be a complete quasi-metric space and let $T: X \rightarrow X$ be a mapping satisfying the following condition:

$$
q(T x, T y) \leq h(q(T x, T y), q(x, y)) q(x, y) \quad \forall x, y \in X,
$$

where $h:[0, \infty) \times[0, \infty) \rightarrow[0, \infty)$ is a function such that $h(t, s)<1$ and $\limsup _{n \rightarrow \infty} h\left(t_{n}, s_{n}\right)<1$ provided that $\left\{t_{n}\right\}$ and $\left\{s_{n}\right\} \subset(0,+\infty)$ are two sequences such that $\lim _{n \rightarrow \infty} t_{n}=$ $\lim _{n \rightarrow \infty} s_{n}$. Then $T$ has a unique fixed point in $X$.

Proof. The result follows from Theorem 29 taking into account that $T$ is a $\mathscr{Z}_{q}$-contraction with respect to $\zeta_{U} \in \mathscr{Z}$, where $\zeta_{U}$ is defined by $\zeta_{U}(t, s)=s h(t, s)-t$ for all $s, t \in[0, \infty)$ (see Example 19).

Example 38. The following example is inspired by Remark 3 in Boyd and Wong [9]. Let $X=[0,1] \cup\{2,3,4, \ldots\}$ and let us define

$$
q(x, y)= \begin{cases}0, & \text { if } x=y \\ x-y, & \text { if } x, y \in[0,1], x>y \\ x+y, & \text { otherwise. }\end{cases}
$$

It is apparent that $(X, q)$ is a complete quasi-metric space, but it is not a metric space (for instance, $q(1,2) \neq q(2,1)$ ). Let us consider the mappings $T: X \rightarrow X, \eta:[0, \infty) \rightarrow \mathbb{R}$, and $\zeta:[0, \infty) \times[0, \infty) \rightarrow \mathbb{R}$ defined by

$$
\begin{gathered}
T x= \begin{cases}x-\frac{1}{2} x^{2}, & \text { if } x \in[0,1], \\
x-1, & \text { if } x \in\{2,3,4, \ldots\},\end{cases} \\
\eta(t)= \begin{cases}t-\frac{1}{4} t^{2}, & \text { if } t \in[0,2], \\
t-\frac{1}{2}, & \text { if } t>2,\end{cases} \\
\zeta(t, s)=\eta(s)-t, \quad \forall t, s \geq 0 .
\end{gathered}
$$

Although $\eta$ is not an upper semicontinuous mapping, it is easy to show that $\zeta$ is a simulation function (if $\left\{t_{n}\right\} \rightarrow \delta>0$ and $\left\{s_{n}\right\} \rightarrow \delta$, then $\lim \sup _{n \rightarrow \infty} \zeta\left(t_{n}, s_{n}\right) \leq \max \left(-\delta^{2} / 4\right.$, $-1 / 2)<0$ ). Furthermore, it can be proved that

$$
\begin{aligned}
& \zeta(q(T x, T y), q(x, y)) \\
& = \begin{cases}\frac{0,}{\frac{(x+3 y)(x-y)}{4},} & \text { if } x, y \in[0,1], x>y, \\
\frac{(x-y)^{2}}{4}, & \text { if } x, y \in[0,1], x<y, \\
\frac{1+y(4-y)}{2}, & \text { if } x=2,0<y \leq 1, \\
\frac{1+x^{2}}{2}, & \text { if } y=2,0<x \leq 1, \\
\frac{1+y^{2}}{2}, & \text { if } x \in\{3,4,5, \ldots\}, y \in[0,1], \\
\frac{1+x^{2}}{2}, & \text { if } y \in\{3,4,5, \ldots\}, x \in[0,1], \\
\frac{3}{2}, & \text { if } x, y \in\{2,3,4, \ldots\}, x \neq y .\end{cases}
\end{aligned}
$$

Therefore, $T$ is a $\mathscr{Z}_{q}$-contraction with respect to $\zeta$. Using Theorem 29, $T$ has a unique fixed point, which is $x=0$.

As Boyd and Wong pointed out in [9], as

$$
\lim _{n \rightarrow \infty} \frac{q(T n, 0)}{q(n, 0)}=\lim _{n \rightarrow \infty} \frac{T n}{n}=\lim _{n \rightarrow \infty} \frac{n-1}{n}=1,
$$

there can be no decreasing function $\varphi$ with $\varphi(t)<1$ for $t>0$ and for which (58) holds. Furthermore, since

$$
\begin{aligned}
\lim _{x \rightarrow 0^{+}} \frac{q(T x, 0)}{q(x, 0)} & =\lim _{x \rightarrow 0^{+}} \frac{T x}{x}=\lim _{x \rightarrow 0^{+}} \frac{x-x^{2} / 2}{x} \\
& =\lim _{x \rightarrow 0^{+}}\left(1-\frac{x}{2}\right)=1,
\end{aligned}
$$

there is no increasing function $\varphi$ with $\varphi(t)<1$ for $t>0$ and for which (58) holds.

Example 39. Let $X=[0, \infty$ ) (it is also possible to consider $X=[0, A]$, where $A>0)$ and let us define

$$
q(x, y)= \begin{cases}x-y, & \text { if } x \geq y \\ \frac{y-x}{2}, & \text { if } x<y .\end{cases}
$$

It is clear that $(X, q)$ is a complete quasi-metric space, but it is not a metric space since $q(1,2) \neq q(2,1)$. Let us define $T$ : $X \rightarrow X$ and $\zeta:[0, \infty) \times[0, \infty) \rightarrow \mathbb{R}$ by

$$
\begin{gathered}
T x=\log (x+1), \quad \forall x \in X \\
\zeta(t, s)=\log (s+1)-t, \quad \forall t, s \geq 0 .
\end{gathered}
$$

Then $\zeta \in \mathscr{Z}$ and $T$ is a $\mathscr{Z}_{q}$-contraction with respect to $\zeta$. Therefore, $T$ has a unique fixed point, which is $x=0$. 


\section{Consequences: Fixed Point Results in the Context of G-Metric Spaces}

In this section, we show the applicability of our main results to the framework of $G$-metric spaces, and we indicate that some existing fixed point results in that setting can be easily derived from our main theorems. First, we recall some basic definitions and fundamental results on this topic which can be found in the literature.

Definition 40 (Mustafa and Sims [3]). A generalized metric (or a $G$-metric) on a nonempty set $X$ is a mapping $G: X \times$ $X \times X \rightarrow[0, \infty)$ satisfying the following properties for all $x, y, z, a \in X$ :

$\left(G_{1}\right) G(x, y, z)=0$ if $x=y=z$;

$\left(G_{2}\right) 0<G(x, x, y)$ for all $x, y \in X$ with $x \neq y$;

$\left(G_{3}\right) G(x, x, y) \leq G(x, y, z)$ for all $x, y, z \in X$ with $y \neq z$;

$\left(G_{4}\right) G(x, y, z)=G(x, z, y)=G(y, z, x)=\cdots$ (symmetry in all three variables);

$\left(G_{5}\right) G(x, y, z) \leq G(x, a, a)+G(a, y, z)$ (rectangle inequality).

In such a case, the pair $(X, G)$ is called a $G$-metric space.

The following result gives some examples of well-known $G$-metrics.

Lemma 41. If $(X, d)$ is a metric space and we define $G_{\text {max }}, G_{\text {sum }}: X \times X \times X \rightarrow[0,+\infty)$, for all $x, y, z \in X$, by

$$
\begin{gathered}
G_{\max }(x, y, z)=\max \{d(x, y), d(y, z), d(z, x)\}, \\
G_{\text {sum }}(x, y, z)=d(x, y)+d(y, z)+d(z, x),
\end{gathered}
$$

then $G_{\max }$ and $G_{\text {sum }}$ are $G$-metrics on $X$.

Example 42. Let $X=[0, \infty)$. The function $G: X \times X \times X \rightarrow$ $[0,+\infty)$, defined by

$$
G(x, y, z)=|x-y|+|y-z|+|z-x|,
$$

for all $x, y, z \in X$, is a $G$-metric on $X$.

Conversely, a $G$-metric always induces quasi-metrics and also metrics.

Lemma 43. Let $(X, G)$ be a $G$-metric space and let us define $q_{G}, q_{G}^{\prime}, d_{G}^{\mathrm{m}}, d_{G}^{\mathrm{s}}: X \times X \rightarrow[0, \infty)$, for all $x, y \in X$, by

$$
\begin{gathered}
q_{G}(x, y)=G(x, x, y), \quad q_{G}^{\prime}(x, y)=G(x, y, y), \\
d_{G}^{\mathrm{m}}(x, y)=\max \{G(x, x, y), G(x, y, y)\}, \\
d_{G}^{\mathrm{s}}(x, y)=G(x, x, y)+G(x, y, y) .
\end{gathered}
$$

Then $q_{G}$ and $q_{G}^{\prime}$ are quasi-metrics on $X$ and $d_{G}^{\mathrm{m}}$ and $d_{G}^{\mathrm{s}}$ are metrics on $X$.

The notions of convergence, Cauchy sequence, and completeness in a $G$-metric space are as follows.
Definition 44. Let $(X, G)$ be a $G$-metric space, and let $\left\{x_{n}\right\}$ be a sequence of points of $X$. We say that $\left\{x_{n}\right\}$ is $G$-convergent to $x \in X$ if

$$
\lim _{n, m \rightarrow \infty} G\left(x, x_{n}, x_{m}\right)=0
$$

that is, for any $\varepsilon>0$, there exists $N \in \mathbb{N}$ such that $G\left(x, x_{n}, x_{m}\right)<\varepsilon$, for all $n, m \geq N$. We call $x$ the limit of the sequence and write $\left\{x_{n}\right\} \rightarrow x$ or $\lim _{n \rightarrow \infty} x_{n}=x$.

Proposition 45. If $(X, G)$ is a G-metric space, then the following statements are equivalent:

(1) $\left\{x_{n}\right\}$ is G-convergent to $x$;

(2) $\left\{G\left(x_{n}, x_{n}, x\right)\right\} \rightarrow 0$ as $n \rightarrow \infty$;

(3) $\left\{G\left(x_{n}, x, x\right)\right\} \rightarrow 0$ as $n \rightarrow \infty$.

Definition 46. Let $(X, G)$ be a $G$-metric space. A sequence $\left\{x_{n}\right\}$ is called a $G$-Cauchy sequence if, for any $\varepsilon>0$, there exists $N \in \mathbb{N}$ such that $G\left(x_{n}, x_{m}, x_{l}\right)<\varepsilon$ for all $m, n, l \geq N$; that is, $\left\{G\left(x_{n}, x_{m}, x_{l}\right)\right\} \rightarrow 0$ as $n, m, l \rightarrow+\infty$.

Proposition 47. Let $(X, G)$ be a $G$-metric space. Then the following are equivalent:

(1) the sequence $\left\{x_{n}\right\}$ is G-Cauchy,

(2) for any $\varepsilon>0$, there exists $N \in \mathbb{N}$ such that $G\left(x_{n}, x_{m}, x_{m}\right)<\varepsilon$, for all $m, n \geq N$.

Definition 48. A $G$-metric space $(X, G)$ is called $G$-complete if every $G$-Cauchy sequence is $G$-convergent in $(X, G)$.

For more details on $G$-metric space, we refer, for example, to $[3,10,11]$.

Lemma 49 (Agarwal et al. [12]). Let $(X, G)$ be a G-metric space and let us consider the quasi-metrics $q_{G}$ and $q_{G}^{\prime}$ as in Lemma 43. Then the following statements hold.

(1) $q_{G}(x, y) \leq 2 q_{G}^{\prime}(x, y) \leq 4 q_{G}(x, y)$ for all $x, y \in X$.

(2) In $\left(X, q_{G}\right)$ and in $\left(X, q_{G}^{\prime}\right)$, a sequence is rightconvergent (resp., left-convergent) if and only if it is convergent. In such a case, its right-limit, its left-limit, and its limit coincide.

(3) In $\left(X, q_{G}\right)$ and in $\left(X, q_{G}^{\prime}\right)$, a sequence is right-Cauchy (resp., left-Cauchy) if and only if it is Cauchy.

(4) In $\left(X, q_{G}\right)$ and in $\left(X, q_{G}^{\prime}\right)$, every right-convergent (resp., left-convergent) sequence has a unique rightlimit (resp., left-limit).

(5) If $\left\{x_{n}\right\} \subseteq X$ and $x \in X$, then $\left\{x_{n}\right\} \stackrel{G}{\rightarrow} x \Leftrightarrow\left\{x_{n}\right\} \stackrel{q_{G}}{\longrightarrow}$ $x \Leftrightarrow\left\{x_{n}\right\} \stackrel{q_{G}^{\prime}}{\longrightarrow} x$.

(6) If $\left\{x_{n}\right\} \subseteq X$, then $\left\{x_{n}\right\}$ is G-Cauchy $\Leftrightarrow \quad\left\{x_{n}\right\}$ is $q_{G^{-}}$ Cauchy $\Leftrightarrow\left\{x_{n}\right\}$ is $q_{G}^{\prime}$-Cauchy.

(7) $(X, G)$ is complete $\Leftrightarrow\left(X, q_{G}\right)$ is complete $\Leftrightarrow\left(X, q_{G}^{\prime}\right)$ is complete.

We present the following version of Theorem 29 in the context of $G$-metric spaces, using the quasi-metric $q_{G}$ defined in Lemma 43. 
Corollary 50. Let $(X, G)$ be a complete G-metric space and let $T: X \rightarrow X$ be a mapping such that there exists $\zeta \in \mathscr{Z}$ verifying

$$
\zeta(G(T x, T y, T y), G(x, y, y)) \geq 0, \quad \forall x, y \in X .
$$

Then $T$ has a unique fixed point in X. Furthermore, every Picard sequence generated by $T$ converges to the unique fixed point of $T$.

Proof. Since $(X, G)$ is complete, then item 7 of Lemma 49 guarantees that $\left(X, q_{G}\right)$ is a complete quasi-metric space, and $T$ is a $\mathscr{Z}_{q_{G}}$-contraction in $\left(X, q_{G}\right)$ with respect to $\zeta$.

The following results are consequence of Corollaries 3037 applied to the quasi-metric $q_{G}(x, y)=G(x, x, y)$ for all $x, y \in X$ (generated by a $G$-metric).

Corollary 51. Let $(X, G)$ be a complete $G$-metric space and let $T: X \rightarrow X$ be a mapping satisfying the following condition:

$$
G(T x, T y, T y) \leq \lambda G(x, y, y), \quad \forall x, y \in X,
$$

where $\lambda \in[0,1)$. Then $T$ has a unique fixed point in $X$.

Corollary 52 (see, e.g., [13]). Let $(X, G)$ be a complete $G$ metric space and let $T: X \rightarrow X$ be a mapping satisfying the following condition:

$$
G(T x, T y, T y) \leq G(x, y, y)-\varphi(G(x, y, y)), \quad \forall x, y \in X,
$$

where $\varphi:[0, \infty) \rightarrow[0, \infty)$ is lower semicontinuous function and $\varphi^{-1}(0)=\{0\}$. Then $T$ has a unique fixed point in $X$.

Corollary 53 (see, e.g., [14]). Let $(X, G)$ be a complete Gmetric space and let $T: X \rightarrow X$ be a mapping. Suppose that, for every $x, y \in X$,

$$
G(T x, T y, T y) \leq \varphi(G(x, y, y)) G(x, y, y)
$$

for all $x, y \in X$, where $\varphi:[0,+\infty) \rightarrow[0,1)$ is a mapping such that $\limsup _{t \rightarrow r^{+}} \varphi(t)<1$, for all $r>0$. Then $T$ has a unique fixed point.

Corollary 54 (cf. [15]). Let $(X, G)$ be a complete G-metric space and let $T: X \rightarrow X$ be a mapping. Suppose that, for every $x, y \in X$,

$$
G(T x, T y, T y) \leq \eta(G(x, y, y))
$$

for all $x, y \in X$, where $\eta:[0,+\infty) \rightarrow[0,+\infty)$ is an upper semicontinuous mapping such that $\eta(t)<t$ for all $t>0$ and $\eta(0)=0$. Then $T$ has a unique fixed point.

Corollary 55. Let $(X, G)$ be a complete $G$-metric space and let $T: X \rightarrow X$ be a mapping satisfying the following condition:

$$
\int_{0}^{G(T x, T y, T y)} \phi(t) q t \leq G(x, y, y), \quad \forall x, y \in X,
$$

where $\varphi:[0, \infty) \rightarrow[0, \infty)$ is a function such that $\int_{0}^{\epsilon} \phi(t) q t$ exists and $\int_{0}^{\epsilon} \phi(t) q t>\epsilon$, for each $\epsilon>0$. Then $T$ has a unique fixed point in $X$.
Finally, we point out that, obviously, if we replace $G(T x, T y, T y)$ and $G(x, y, y)$ in Corollaries $50-55$ by the expressions $G(T x, T y, T z)$ and $G(x, y, z)$, respectively, then the conclusion is still valid (because the contractive conditions are stronger).

\section{Conflict of Interests}

The authors declare that there is no conflict of interests regarding the publication of this paper.

\section{Authors' Contribution}

All authors contributed equally and significantly in writing this paper. All authors read and approved the final paper.

\section{Acknowledgments}

This research was supported by Deanship of Scientific Research (DSR), King Abdulaziz University, Jeddah, Saudi Arabia. The authors thank the anonymous referees for their remarkable comments, suggestions, and ideas that helped to improve this paper.

\section{References}

[1] M. Jleli and B. Samet, "Remarks on G-metric spaces and fixed point theorems," Fixed Point Theory and Applications, vol. 2012, article 210, 2012.

[2] B. Samet, C. Vetro, and F. Vetro, "Remarks on G-metric spaces," International Journal of Analysis, vol. 2013, Article ID 917158, 6 pages, 2013.

[3] Z. Mustafa and B. Sims, "A new approach to generalized metric spaces," Journal of Nonlinear and Convex Analysis, vol. 7, no. 2, pp. 289-297, 2006.

[4] F. Khojasteh, S. Shukla, and S. Radenović, "A new approach to the study of fixed point theorems via simulation functions," Filomat. In press.

[5] M. S. Khan, M. Swaleh, and S. Sessa, "Fixed point theorems by altering distances between the points," Bulletin of the Australian Mathematical Society, vol. 30, no. 1, pp. 1-9, 1984.

[6] F. E. Browder and W. V. Petryshyn, "The solution by iteration of nonlinear functional equations in Banach spaces," Bulletin of the American Mathematical Society, vol. 72, pp. 571-575, 1966.

[7] S. Banach, "Sur les opérations dans les ensembles abstraits et leur application auxéquations intégrales," Fundamenta Mathematicae, vol. 3, pp. 133-181, 1922.

[8] B. E. Rhoades, "Some theorems on weakly contractive maps," Nonlinear Analysis: Theory, Methods \& Applications, vol. 47, pp. 2683-2693, 2001.

[9] D. W. Boyd and J. S. W. Wong, "On nonlinear contractions," Proceedings of the American Mathematical Society, vol. 20, no. 2, pp. 458-464, 1969.

[10] Z. Mustafa and B. Sims, "Fixed point theorems for contractive mappings in complete G-metric spaces," Fixed Point Theory and Applications, vol. 2009, Article ID 917175, 10 pages, 2009.

[11] Z. Mustafa, A new structure for generalized metric spaces with applications to fixed point theory [Ph.D. thesis], The University of Newcastle, Callaghan, Australia, 2005. 
[12] R. Agarwal, E. Karapınar, and A. F. Roldán-López-de-Hierro, "Fixed point theorems in quasi-metric spaces and applications to coupled/tripled fixed points on $\mathrm{G}^{*}$-metric spaces," Journal of Nonlinear and Convex Analysis. In press.

[13] C. T. Aage and J. N. Salunke, "Fixed points for weak contractions in G-metric spaces," Applied Mathematics E-Notes, vol. 12, pp. 23-28, 2012.

[14] R. P. Agarwal and E. Karapınar, "Remarks on some coupled fixed point theorems in G-metric spaces," Fixed Point Theory and Applications, vol. 2013, article 2, 33 pages, 2013.

[15] W. Shatanawi, "Fixed point theory for contractive mappings satisfying $\phi$-maps in G-metric spaces," Fixed Point Theory and Applications, vol. 2010, Article ID 181650, 9 pages, 2010. 


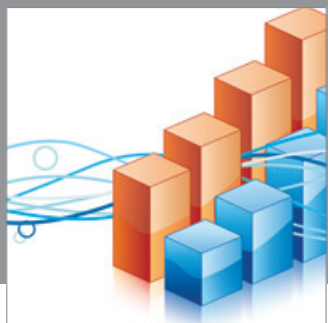

Advances in

Operations Research

mansans

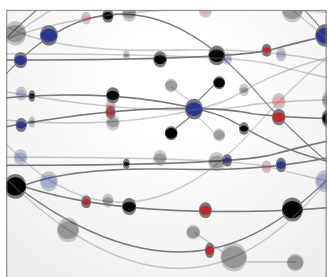

The Scientific World Journal
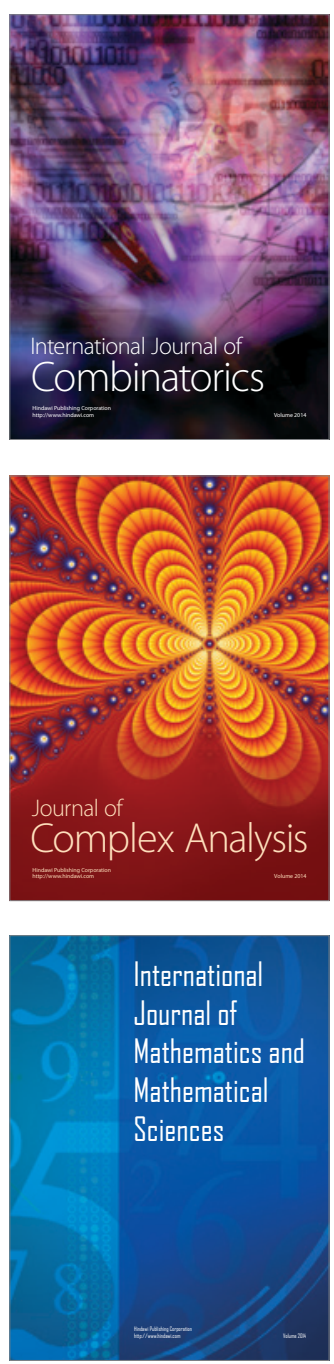
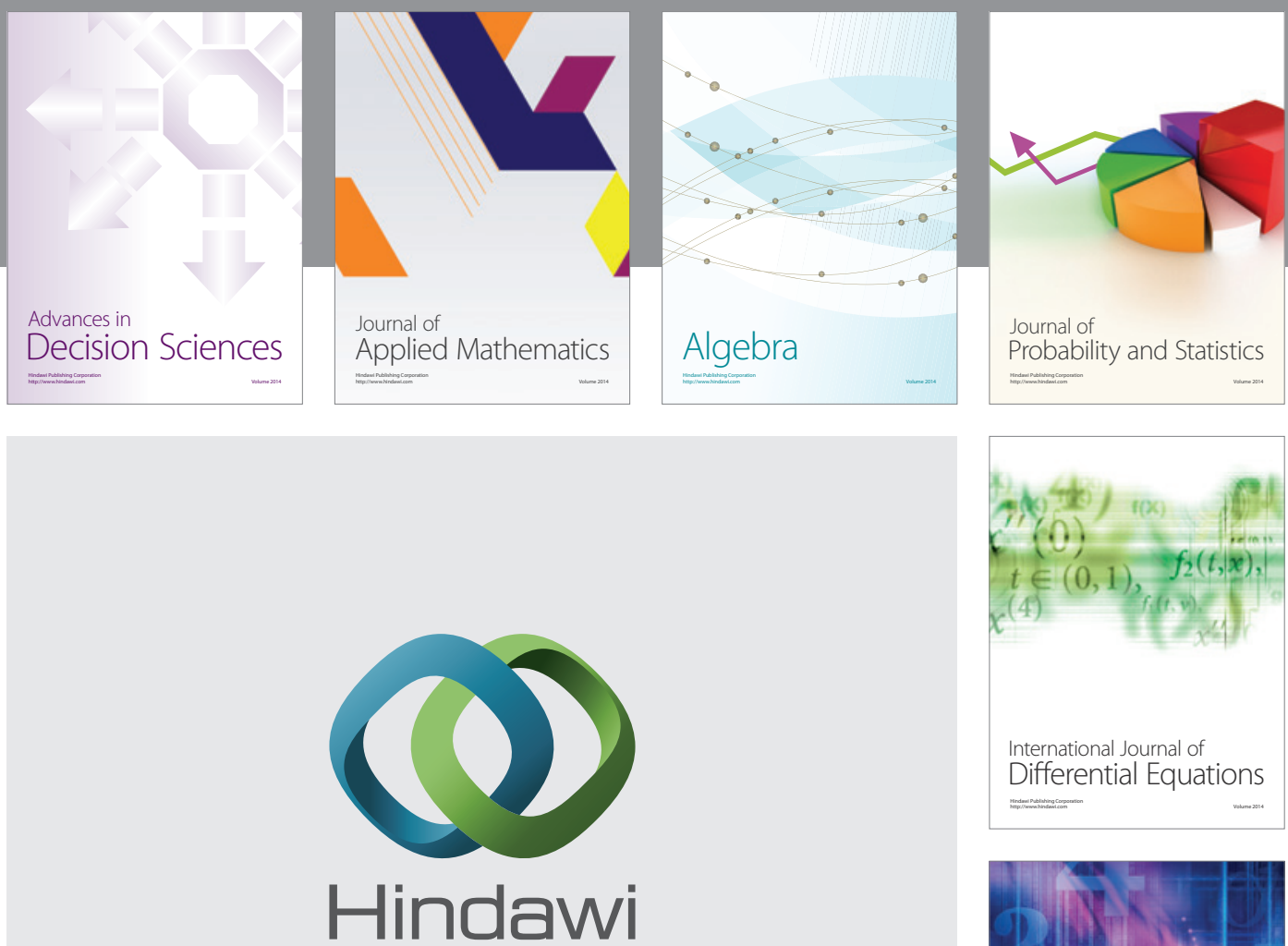

Submit your manuscripts at http://www.hindawi.com
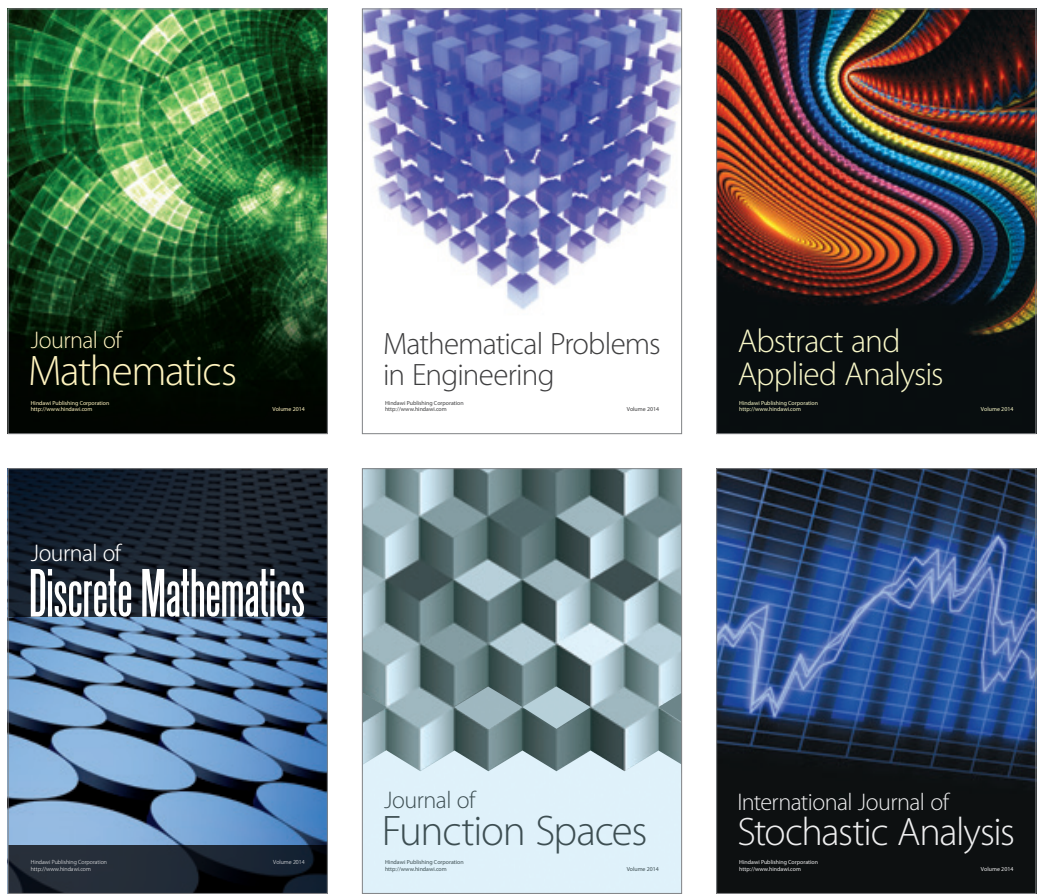

Journal of

Function Spaces

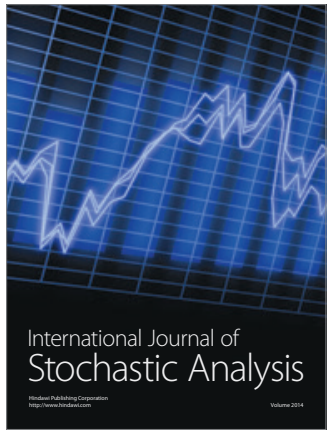

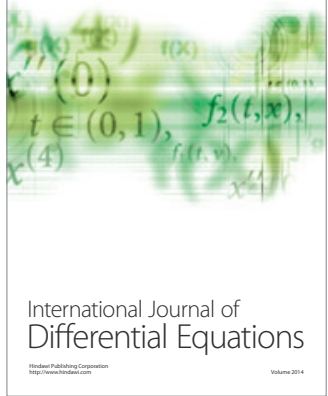
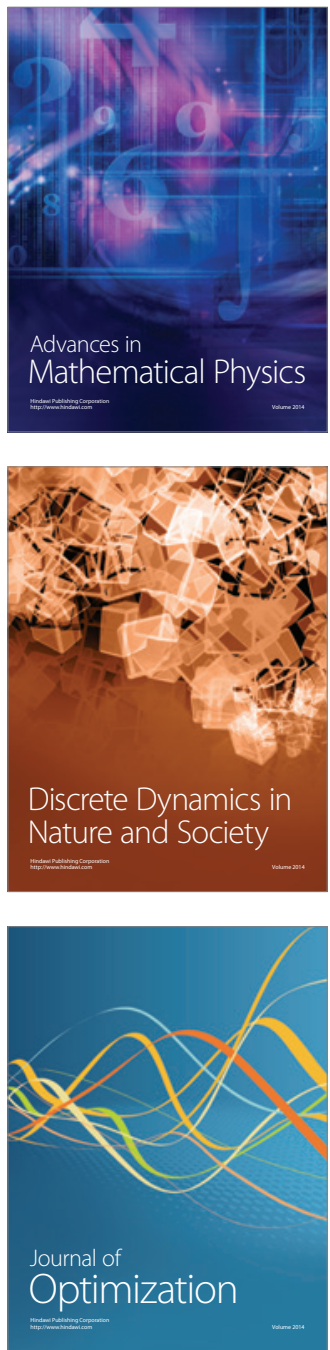